Planetary Systems in the Universe - Observation, Formation and Evolution

Proceedings IAU Symposium No. 202, (C)2004 IAU

Alan Penny, Pawel Artymowicz, Anne-Marie Lagrange, \& Sara Russell, eds.

\title{
Planetary mass limits using Hipparcos astrometry
}

\author{
Frédéric Arenou and Julienne Palasi \\ CNRS/Observatoire de Paris, France
}

\begin{abstract}
Using Hipparcos and Tycho-2 data, together with the radial velocity information, a statistical analysis is applied to all the orbital solutions of planetary candidates, in order to avoid a bias towards small inclinations. There are hints of additional, longer period companions.
\end{abstract}

\section{Introduction}

Since Perryman et al. (1996) who gave an upper limit to the mass of 3 planetary companions of main-sequence stars, the Hipparcos astrometry has been used to get an estimate of the mass of the outermost planet of $v$ And (Mazeh 1999) and to reject the HD 10697 companion into the brown dwarf region (Zucker \& Mazeh 2000).

Hipparcos astrometric data do not allow by itself to detect low-mass companions, except perhaps those orbiting nearby stars with a long period. However, the combination of radial velocity data with the astrometric data may give an estimate of the reflex semi-major axis ( $a_{1}$, in milliarcsec), and thus of the secondary mass. We have computed an orbital solution for known stars with planetary companions, although the reflex motion is presumably much smaller than the astrometric precision, with the following motivations:

- there are indications that combining the radial velocity orbital parameters of planetary candidates to the Hipparcos data may give masses biased towards unreasonably large values if the error law of the orbital parameters is not accounted for;

- inadequate orbital solutions may give a hint of additional companions;

- a statistical analysis allows to study the unprojected $M_{2}$ distribution.

\section{Combined orbital solutions for planetary candidates}

One-dimensional abscissae residuals have been extracted from the Hipparcos Intermediate Astrometric Data. The radial velocity orbital parameters $(P, T, e$, $\left.\omega_{1}, a_{1} \sin i\right)$, are introduced as observations, with their weight, and a full astrometric+orbital solution is computed by least-square. We also used Tycho-2 (Høg et al., 2000) proper motions, when available, as supplementary observations, since this may help to detect longer period companions, or to improve the astrometric and semi-major axis precision. For example, the use of Hipparcos+Tycho- 
2 for the putative brown dwarf HD 127506 (Halbwachs et al. 2000) improves the mass precision, $M_{2}=39.2 \pm 9.9 \mathrm{M}_{\mathrm{Jup}}$ thus confirming its brown dwarf status.

This study is limited to 31 planetary candidates, extracted from the Extrasolar planet Encyclopedia (Schneider, 2000), for which a solution could be found. The computed semi-major axis cannot however be taken at face value for several reasons, related to the way the data analysis is performed. The first problem occurs in the case where there are astrometric perturbations due to some longer period companion, since the astrometric residuals, and thus the computed $a_{1}$, will merely reflect the influence of this companion, instead of the short period one.

The other pitfall has been shown in Halbwachs et al. (2000). When the true semi-major axis is small compared to the astrometric precision, the computed semi-major axis follows a law similar to a Rayleigh-Rice (RR) law. Consequently up to $a_{1} / \sigma_{a_{1}} \approx 3$, the computed $a_{1, \text { obs }}$ will be larger than the true value on the average, so unreasonably small inclinations (and large masses) may be obtained, since $a_{1} \sin i$ is constrained to a small value by the radial velocity analysis.

For instance, Mazeh et al. (1999) obtained a semi-major axis $a_{1, \mathrm{obs}}=$ $1.4 \pm 0.6$ mas for $v$ And; simulations of Hipparcos abscissae show that on the average this value may be overestimated by about $15 \%$. For other stars with smaller $a_{1} / \sigma$ the overestimation is much more larger.

Due to the $a_{1} \sin i$ constraint, the error law of the inclination is worse: as a typical example, the inclination of $\mathrm{HD} 209458$ is found formally to be $i=179.95 \pm 0.05^{\circ}$, whereas the true value is $i=86.1 \pm 1.6^{\circ}$ (Mazeh et al., 2000). For a large number of planetary companions such small, and statistically unlikely $\sin i$ are derived, due to the RR law.

Even when the $a_{1, \mathrm{obs}} / \sigma$ is significant, a conclusion cannot always be easily drawn. Consider for instance HD 195019, where the computed semi-major axis is $a_{1, \mathrm{obs}}=2.75 \pm 0.59$ mas. Taking this result at face value would however imply a secondary as massive as the primary, and a $0.26^{\circ}$ inclination. This value has an unrealistic $10^{-5}$ probability if the inclinations are random.

In front of these various problems, it is then statistically more sensible to give only an upper limit to the mass of the companion. In most cases, no valuable information can be derived since the lower mass limit will be in the planetary domain while the upper limit is in the brown dwarf or stellar domain. However there are 4 stars for which the Hipparcos+Tycho- 2 astrometric data assess at more than $1 \sigma$ that the upper limit of the secondary mass is below 15 Jup: $v$ And, 47 UMa, 14 Her and 16 Cyg B.

\section{Posterior planetary mass distribution}

Assuming as likelihood a RR error law $f\left(a_{1, \mathrm{obs}} \mid a_{1}\right)$ for the observed semi-major axis, a bayesian analysis has been performed. Noting $A=\pi a_{1} \sin i$ given by spectroscopy, with $f(i)=\sin i$ in the case of random inclinations, then $f\left(a_{1} \mid A\right)=$ $A^{2} / a_{1}^{2} \sqrt{a_{1}^{2}-A^{2}}$ for $a_{1}>A$ and 0 otherwise. A posterior pdf $f\left(a_{1} \mid a_{1, \mathrm{obs}}, A\right)$ is found for each star, then the cumulative distribution is computed and a $95 \%$ posterior confidence interval for $M_{2}$ is finally estimated, taking also into account 
the $M_{1}$ error bar. For example we obtain $[4,13] \mathrm{M}_{\mathrm{Jup}}$ for the outermost planet of $v$ And and [6, 42] $\mathrm{M}_{\mathrm{Jup}}$ for HD 10697, almost, although not completely assessing the brown dwarf versus planetary hypothesis.

Concerning the whole sample, the distribution of the posterior median planetary masses is statistically consistent with a $1 / M_{2}$ law. After a convolution by a $\sin i$ law, the distribution of the projected posterior median masses is however not consistent ( $2 \%$ probability) with the observed $M_{2} \sin i$ distribution. The reasons are that the RR law is not fully representative of the $a_{1, \mathrm{obs}}$ error law, and also that additional, more massive companions may perturb the astrometric solution.

\section{Hint for additional companions}

Astrometry is more sensitive to longer periods, and long term proper motions have been used in our solutions. So that if a long period and more massive companion is present, the adopted orbital model with one companion only should prove to be inadequate.

In a recent paper, Fischer et al. (2000) show that $\tau$ Boo, $55 \mathrm{Cnc}$, HD 217107 and HD 38529 exhibit residual radial velocities consistent with additional companions. Our solution for Tau Boo has a (gaussian) goodness of fit GOF $>6$, in support to Fischer et al. findings, who attribute the residual RV trend to a M2V companion. HD 38529 has already an 'acceleration solution' in the Hipparcos Catalogue, and the orbital solution has a GOF=2, suggesting the presence of another companion. The GOF is however about 0 for $55 \mathrm{Cnc}$ and HD 217107 which would then favor a light secondary mass if an additional companion is present. Two other stars may be concerned: HD 46375 has a large GOF, and Gl 86 with GOF=19 confirms that the long term RV trend (Queloz et al., 2000) may be due to a more massive companion.

\section{References}

Fischer, D. et al. 2000, ApJ, submitted

Halbwachs, J.L., Arenou, F., Mayor, M., Udry, S., Queloz, D. 2000,A\&A355,581

$\mathrm{H} \varnothing \mathrm{g}, \mathrm{E}$, et al. 2000, A\&A 355, 27

Mazeh, T., Zucker, S., dalla Torre, A., van Leeuwen, F. 1999, ApJ 522, L149

Mazeh, T., et al., 2000, ApJ 532, 55

Perryman, M.A.C. et al. 1996, A\&A 310, L21

Queloz, D. et al. 2000, A\&A 354, 99

Schneider, J. 2000, http://www.obspm.fr/planets , 1st August version

Zucker, S., Mazeh, T. 2000, ApJ 531, 67 